\title{
Le rôle des religieuses hospitalières de Saint-Joseph dans \\ l'éducation au Madawaska depuis 1873
}

\section{Georgette Desjardins}

Volume 48, 1981

URI : https://id.erudit.org/iderudit/1007100ar

DOI : https://doi.org/10.7202/1007100ar

Aller au sommaire du numéro

Éditeur(s)

Les Éditions Historia Ecclesiæ Catholicæ Canadensis Inc.

ISSN

0318-6172 (imprimé)

1927-7067 (numérique)

Découvrir la revue

Citer cet article

Desjardins, G. (1981). Le rôle des religieuses hospitalières de Saint-Joseph dans l'éducation au Madawaska depuis 1873. Sessions d'étude - Société canadienne d'histoire de l'Église catholique, 48, 57-66. https://doi.org/10.7202/1007100ar

Tous droits réservés @ Les Éditions Historia Ecclesiæ Catholicæ Canadensis Inc., 1981
Ce document est protégé par la loi sur le droit d'auteur. L'utilisation des services d'Érudit (y compris la reproduction) est assujettie à sa politique d'utilisation que vous pouvez consulter en ligne.

https://apropos.erudit.org/fr/usagers/politique-dutilisation/ 


\section{Le rôle des religieuses hospitalières de Saint-Joseph dans l'éducation au Madawaska depuis 1873}

En octobre 1873, sept soeurs de l'Hôtel-Dieu quittent Montréal pour venir ouvrir une mission au Madawaska, Nouveau-Brunswick. Les Religieuses Hospitalières de Saint-Joseph s'aventurent alors « hors des sentiers battus", car pour la première fois dans l'histoire de leur Institut, elles s'engagent à s'occuper d'abord de l'éducation ; le soin des malades viendra en deuxième lieu. Ceci parce que le couvent qu'elles habiteront est construit sur un terrain légué en 1857 par le curé Antoine Langevin pour l'établissement d'une école destinée à l'éducation des jeunes filles de Madawaska.

Pour quels motifs les religieuses de Montréal acceptent-elles, en 1873, cette mission qui oblige des Hospitalières à devenir enseignantes ? Qui est l'initiateur de ce projet? L'annaliste de l'Hôtel-Dieu de SaintBasile écrit vers 1910 :

Tout nous porte à croire que ce fut notre Mère Davignon, alors supérieure à Chatham, qui ... conçut la première l'idée d'une nouvelle fondation à Saint-Basile de Madawaska. Ce qui l'engageait à poursuivre ce dessein, c'est que les Soeurs de la Charité qui demeuraient ici depuis près de 15 ans, venaient d'être rappelées au mois de mai, pour des raisons que nous ignorons.

Si le projet se réalise, c'est grâce à $\mathrm{Sr}$ Davignon et à Mère Pagé, supérieure de l'Hôtel-Dieu de Montréal, et non, comme nous l'avons cru jusqu'à tout dernièrement, à l'évêque de Chatham. En effet, $\mathbf{M}^{\mathrm{gr}}$ James Rogers n'entre pas d'emblée dans les vues de $\mathbf{S}^{\mathrm{r}}$ Davignon même s'il désire la réouverture de l'Académie de Madawaska laissée vacante par le départ des Soeurs de la Charité de Saint-Jean. $\mathbf{M}^{\mathrm{gr}}$ appréhende ne pouvoir subvenir aux besoins spirituels et temporels d'une communauté religieuse cloîtrée établie au lointain Madawaska. Il donne néanmoins sa bénédiction au projet des Religieuses Hospitalières et le soutient financièrement et moralement quoique, selon les Annales, il "paraissait ne vouloir pas trop se mêler de la Fondation disant qu'elle n'était pas son ouvrage ". 
Cette nouvelle mission des Soeurs de l'Hôtel-Dieu de Montréal est bien l'ouvrage d'une religieuse trop peu connue à Saint-Basile. Il y aurait beaucoup à dire sur $\mathrm{S}$ L Louise-Virginie Davignon, femme de foi audacieuse qui, bien que gravement atteinte par le cancer, s'offre pour la mission difficile du Madawaska où elle mourra, âgée de 50 ans seulement, quatre mois à peine après son arrivée. Ce billet qu'elle écrit à Mère Pagé, peu avant sa mort, révèle un peu les qualités de coeur et d'esprit de Sr Davignon :

Ne regrettez pas, ma Mère, de m'avoir envoyée l'automne dernier. Dieu a èu ses desseins, car l'hôpital aurait peut-être été de longues années sans se bâtir. S'il y a eu faute en nous pressant d'accepter cette fondation, c'est moi qui suis coupable, mais heureuse faute, chère Mère, qui me procure beaucoup de consolation à la mort. Encore une fois n'ayez aucun regret.

Pourquoi Soeur Davignon désire-t-elle voir sa congrégation s'implanter au Madawaska ? Elle et Mère Pagé savent que la pauvreté du milieu et la Loi des Écoles non-confessionnelles de 1871 sont les principales causes du départ des Soeurs de la Charité qui ont dirigé l'Académie de Madawaska de 1859 à 1873 . De plus, en cet automne de 1873, rien ne laisse envisager la fin de la crise économique en Amérique et l'assouplissement, au Nouveau-Brunswick, de la Loi des Écoles neutres. Or, c'est précisément parce que le Madawaska est démuni de services essentiels que les Religieuses Hospitalières veulent venir s'y établir. D'ailleurs, un couvent est vide et prêt à accueillir une nouvelle communauté, pense-t-on à Montréal. Les Soeurs ne savent cependant pas que leur venue est peu désirée par les "notables" de la région qui craignent d'être trop sollicités par ces religieuses cloîtrées. Les annales de l'Hôtel-Dieu de Saint-Basile laissent clairement entendre que les premiers bienfaiteurs de l'oeuvre sont les humbles et les pauvres. Les prêtres et les " grands " invitent plutôt les Soeurs à retourner à Montréal.

Malgré l'accueil très froid qui leur est réservé, les Hospitalières sont vite sollicitées d'ouvrir le pensionnat le plus tôt possible et on demande, en plus, l'enseignement de l'anglais et de la musique. Les Soeurs ne s'attendent pas à ces exigences si l'on en croit une lettre du 20 octobre 1873 : " Nos Devancières s'étant plus attachées à l'enseignement anglais, quoique nous soyions ( $\mathrm{sic}$ ) parmi les Canadiens, ils croient ne pas pouvoir se passer de cette langue et ils demandent une Maîtresse. Plusieurs demandent la musique ».

Puisque les Hospitalières ont accepté l'oeuvre de l'Académie, elles se doivent de répondre le mieux possible aux désirs des parents. Le 
7 janvier 1874, les classes reprennent et le Pensionnat accueille une dizaine de jeunes filles. La tâche s'avère très lourde pour ces Hospitalières devenues institutrices et éducatrices ; et l'oeuvre n'est maintenue que grâce à la charité qui unit les Soeurs de Saint-Basile à leurs compagnes de Montréal, Kingston, Chatham et même de France. Ces communautés d'Hospitalières, qui ne sont pas encore unies en Généralat, s'imposent de lourds sacrifices pour venir en aide à la jeune mission du Madawaska, en envoyant soit une maîtresse d'anglais ou de piano, soit des dons en argent et en effets. Les annales commentent brièvement la situation :

Il semblait que le démon déployait toutes les ruses pour semer l'ivraie de la division et empêcher le bien que l'oeuvre était appelée à faire. Chaque jour se présentaient de nouvelles épreuves. Celles qui provenaient du pensionnat n'étaient pas les moindres. Elles étaient obligées d'enseigner les deux langues... Soeur Brissette, qui savait à peine lire l'anglais, se voyait obligée d'essayer à l'enseigner aux enfants. Soeur Perrin ignorait le calcul, science absolument nécessaire. Les élèves s'aperçurent bientôt de la réalité... Les classes se continuaient malgré toutes les difficultés.

Un autre grand problème réside alors dans le recrutement des élèves. En 1874, les écoles publiques, fermées depuis près de trois ans à cause de la loi de 1871, ouvrent graduellement leurs portes. Les parents qui paient des taxes pour ces écoles peuvent difficilement envoyer leurs filles à l'Académie. Ces nouvelles parviennent à Montréal où l'on se demande si, dans ces circonstances, les Religieuses Hospitalières doivent maintenir cette oeuvre ingrate. Sur ces entrefaites, deux personnalités de Madawaska conseillent aux Soeurs de conformer leur enseignement aux exigences provinciales afin de recevoir des salaires et un plus grand numbre d'élèves. Cette proposition plaît à certaines soeurs mais est rejetée par la majorité. Il faut se mettre dans le contexte de l'époque pour comprendre les raisons de ce refus. On n'est pas encore à l'ère de l'oécuménisme, loin de là ! L'annaliste du convent écrit :

Nos Mères préférèrent, pour le moment, vivre plus pauvre et remplir indépendamment d'un gouvernement protestant, leurs devoirs de zèle et de dévouement auprès des malades et des enfants. Il est vrai qu'on les assurait qu'elles seraient libres d'enseigner la religion mais qui pouvait leur promettre au début de cette loi inique qu'elles garderaient leur liberté?

Bien que conscientes de leur incompétence et de leurs limites personnelles et communautaires, les Hospitalières s'efforcent d'être de bonnes éducatrices. Les Annales de l'Hôtel-Dieu nous disent que "des petites séances dramatiques et musicales pour former les enfants 
à la politesse et attirer de l'estime à l'Établissement et par cela même, de l'honneur à la Religion "sont organisées. La première, présentée les 8 et 9 février 1875 , rapporte $\$ 7.50$ en argent et $\$ 3.00$ en effets. Les Soeurs font aussi leur possible pour encourager les jeunes filles qui manifestent beaucoup d'intérêt pour "les ouvrages de goût". Ces éducatrices inexpérimentées encourent cependant la désapprobation et l'indignation de certaines personnes sages qui comprennent mal comment des religieuses aussi pauvres pouvaient se permettre d'acheter du velours, des nerles et de la dentelle. Il faut dire que, par ignorance du coût des articles, une Soeur a placé une commande pour une valeur de plus de $\$ 300$. On croit alors qu'un «petit magasin de campagne " est à la veille d'ouvrir.

Malgré les incompréhensions et les contrariétés nombreuses, l'Académie demeure ouverte de 1874 à 1876. À l'époque, c'était la seule école au Madawaska où se donnait un enseignement au-delà de l'élémentaire. De plus, il n'y avait pas d'autre pensionnat dans la région. Les Soeurs sont désireuses de répondre à un véritable besoin, mais le découragement ébranle la Supérieure et plusieurs de ses compagnes. Puis, à Montréal, Mère Pagé a terminé son mandat de supériorat et elle est remplacée par une Soeur qui ne croit pas en l'avenir des Hospitalières au Madawaska. En outre, à Saint-Basile, quelques religieuses préconisent la fermeture de l'Académie. Cependant, Mère Pagé veille sur l'oeuvre de Soeur Davignon et ne ménage pas ses mots dans une lettre du 10 septembre 1876. Cette femme d'expérience conseille de faire lever l'hôpital sans tarder " sur votre terrain ", dit-elle. Puis elle donne clairement son opinion :

Vous comprenez bien qu'il vous faudra garder l'école encore de longues années, pour la raison bien simple que cette localité, n'étant pas en état de faire vivre une Communauté, ne pourra en faire subsister deux. Ce serait vous exposer à tout perdre si vous cherchiez à bâtir ailleurs à présent, car je sais bien que c'est l'éducation avant tout que Mgr Rogers veut ; si vous l'abandonniez, vous seriez vite remerciées. Ce n'est pas le temps de penser à cela ; pour moi, si j'y étais je n'y penserais jamais, parce que tant que vous aurez l'école, la propriété est à vous, rappelez-vous bien cela. Et pourquoi ne pas faire ce que nos Communautés de France ont fait pendant plus de 200 ans et ce que quelques-unes font encore?

Les Hospitalières à Saint-Basile n'ont pas beaucoup de choix ; elles doivent garder l'Académie ou quitter le milieu. Et à l'automne de 1876, plusieurs préferent abandonner la mission du Madawaska. Or Mère Pagé trouve à Saint-Basile une digne émule de la fondatrice Soeur Davignon. La jeune et dynamique Soeur Alphonsine Ranger, 
dite Soeur Maillet, a foi en l'avenir et elle entreprend des démarches pour empêcher la fermeture du couvent. Le 18 septembre 1876, Soeur Maillet, qui n'a pas encore 30 ans, écrit (avec ou sans l'approbation de sa Supérieure, on ne sait), une longue lettre à Mgr Bourget de Montréal :

La pensée qu'il faudra abandonner ces chères enfants que nous instruisons et ces pauvres malades qui ne peuvent avoir d'autres secours sur la terre que nous, me saigne le coeur... mais la pensée que si Dieu est pour nous rien au monde ne pourra nous renverser, me ranime, et avec cela mon courage s'accroit à proportion des difficultés... Nous serons pauvres longtemps, mais nous imiterons en cela notre Divin Maître et nos premières Mères...

On cite l'ingratitude des gens et le peu d'importance que l'on attache à une éducation chrétienne et au bonheur de posséder une maison religieuse ; je vois bien cela dans un grand nombre, mais c'est pour moi un motif plus pressant de les aimer davantage et de leur faire du bien... Je suis prête à tous les sacrifices pour procurer à ces pauvres âmes le bonheur d'une vie chrétienne. Si nous n'étions pas si pauvres et si j'étais libre de faire ce que je voudrais pour empêcher le mal que produisent ces écoles diaboliques, je demanderais à ce que l'enseignement des externes fût donné gratuitement. Par ce moyen peut-être parviendrions nous à faire honte à nos ennemis ; au moins, leur prouverions-nous que c'est moins pour un gain sordide que nous travaillons, que pour gagner des âmes à Notre-Seigneur...

Ces paroles jaillies du coeur tendre et compréhensif de l'intrépide Soeur Maillet touchent $\mathrm{M}^{\mathrm{gr}}$ Bourget qui, d'ailleurs, a fortement encouragé la fondation en 1873. Il intervient auprès des autorités de la communauté mère de Montréal pour empêcher la fermeture du couvent de Saint-Basile.

La crise des trois premières années s'atténue après 1876 et, avec la reprise économique qui s'amorce en Amérique, une ère de progrès s'annonce au Madawaska. Au printemps de 1879, un grand désir de Soeur Maillet est exaucé : les filles externes seront dorénavant admises gratuitement à l'Académie. Les Soeurs favorisent ainsi l'accès à une meilleure éducation à un plus grand nombre de jeunes filles. De plus, les Hospitalières accordent une attention toute particulière aux orphelins ; en 1884 et 1885 s'ouvrent officiellement deux orphelinats où l'on accepte, souvent gratuitement, 25 fillettes et 25 petits garçons. Ces derniers ont de petits confrères puisque le "Couvent " a maintenant un pensionnat pour garçons. 
L'Académie de Madawaska a. en quelques années, acquis une bonne renommée. Le district scolaire de Saint-Basile demande en 1884 à Soeur Trudel, qui a son diplôme de l'École Normale. de prendre la direction de l'école paroissiale. La communauté accède à cette requête et l'Académie devient une école publique reconnue par la Province. C'est aussi à l'Académie que les jeunes filles et garçons du Nord-Ouest du NouveauBrunswick et même du Maine viennent se préparer pour être admis à l'École Normale.

Le curé de la paroisse. l'abbé Louis-Napoléon Dugal. suit de très près l'enseignement dispensé au couvent. Il donne lui-même des cours. spécialement dans les classes des garçons : on parle même du " petit Collège de $\mathrm{M}^{\mathrm{g} r}$ Dugal ». Les inspecteurs d'école donnent des rapports élogieux sur l'école du couvent. Un extrait d'un rapport de 1888 se lit ainsi :

Il y a trois départements au Couvent de Saint-Basile. le plus avancé étant sous la direction de Soeur Marie Trudel, maitresse d'une grande compétence. L'enseignement est porté à un très haut niveau et la formation morale et intellectuelle est par excellence ce que l'on peut désirer pour ceux qui se préparent aux luttes de la vie.

Tout n'est pas parfait, cependant. S'il y a des compliments, il y a aussi des critiques. Dans les Chroniques du 15 juillet 1890, l'annaliste a noté : "Les Commissaires des Écoles Mess. Hudon et Cyprien Martin, ont écrit à l'inspecteur au sujet des plaintes qui ont été faites sur notre école. M. l'inspecteur O'Brien avait paru cependant très satisfait lors de sa dernière visite ". Quelles sont les causes de mécontentement? Nous n'en savons rien. Quoi qu'il en soit, les Soeurs font leur possible pour remédier à la situation et pour répondre aux attentes des enfants et des parents. Les chroniques nous disent que, le 4 septembre 1894, la Supérieure nomme une Soeur française maîtresse du Pensionnat et lui donne une soeur anglaise pour compagne " parce que les Parents lui demandent que leurs enfants apprennent à parler anglais". Or, l'abbé Dugal désire que les petits garçons aussi apprennent l'anglais. Les deux maîtresses des Pensionnats s'entendent ensemble pour donner satisfaction aux garçons comme aux filles : " La maitresse anglaise gardera tous les deux jours chez les garçons... et la maîtresse française gardera tous les deux jours chez les filles ». L'annaliste ajoute : "L'entreprise était un peu difficile et a exigé bien des sacrifices, mais la charité et l'union qui règnent entre nous font que tout cela se fait sans inconvénients ». 
L'oeuvre des Hospitalières est placée sous la protection de leur saint patron et la dévotion à Saint-Joseph se répand parmi les enfants et les malades et par eux dans la région. Les célèbres pèlerinages du mois de Saint-Joseph, tout particulièrement celui des orphelins, attirent des foules au couvent.

On ne parle plus de fermeture maintenant même si les problèmes surgissent de tous côtés. L'amour et la tendresse que les Hospitalières ont pour les orphelins et les pauvres les obligent à aménager des locaux toujours plus vastes. Les revenus sont insuffisants et les emprunts causent beaucoup de soucis. Cependant, l'oeuvre de l'Hôtel-Dieu continue selon l'esprit de la Congrégation et en conformité avec les buts particuliers de cette maison du Madawaska. Un extrait des Annales de 1910 exprime ce que les Hospitalières s'efforcent de vivre à Saint-Basile :

Nos Mères Fondatrices ont apporté de la Maison-Mère l'amour des pauvres. C'est là l'esprit de notre sainte vocation. En parcourant les annales de la fondation, on voit que dès son berceau. le pauvre y fut accueilli avec bonheur et soigné dans un esprit de charité.

À côté de cette oeuvre essentielle... a grandi aussi une oeuvre accessoire à l'Institut mais qui est l'oeuvre première dans l'HôtelDieu de Saint-Basile : celle de l'éducation de la jeunesse. Nous n'avons qu'à jeter un coup d'oeil sur notre histoire pour voir comme cette oeuvre est unie à l'oeuvre de l'hospitalité : elles ont prospéré l'une près de l'autre comme les rameaux d'une même vigne et ont été alimentées de la même sève, qu'est la charité.

À la fin du $19^{\mathrm{e}}$ siècle, les Religieuses Hospitalières de Saint-Joseph au Madawaska peuvent compter sur l'encouragement et la collaboration du clergé et des laïques dont la bienveillante générosité va tout particulièrement vers les orphelins et les pauvres. Une pionnière, Soeur Maillet, est sans contredit le pilier des oeuvres de l'Hôtel-Dieu depuis le décès de la fondatrice, Soeur Davignon. Le dynamisme et l'esprit de foi de Soeur Maillet rayonnent dans le milieu et la population apprécie ce que les Soeurs font pour venir en aide aux petits et aux faibles. Les bazars annuels sont bien connus et des contributions parviennent d'un peu partout. Une petite rubrique parue le 10 juillet 1888 dans Le Moniteur Acadien révèle l'esprit d'entraide qui règne :

Les 11 et 12 juillet, grand bazar pour venir en aide au nouvel hôpital et à l'orphelinat en voie de construction. Le chemin de fer du N.-B. émettra à cette occasion des billets d'excursion et il est à souhaiter qu'un bon nombre d'étrangers iront prêter main forte aux braves et religieux habitants de Saint-Basile. 
Citons cet autre extrait des chroniques de l'Hôtel-Dieu en date des 17 et 18 juillet 1889 : "Bazar et Pique-nique. $\$ 700$. de profit. Dévouement. générosité et cordiale sympathie de tous et surtout de St-Basile et d'Edmundston ". Le 2 mai 1892, les Indiens du groupe "Docteurs Kickapoo)" apportent \$39.38, bénéfice de deux concerts donnés à Edmundston en faveur des pauvres et des orphelins. L’annaliste ne peut plus écrire comme elle l'a fait pour les années 1873-1876 : "Dieu semblait permettre qu'elles fussent le rebut de tous".

Les premières décennies du 20` siècle n’apportent pas de changements majeurs dans l'oeuvre d'éducation à l'Hôtel-Dieu de Saint-Basile. Par contre, du nouveau s'annonce après la grande crise économique des années 1930. D'autres femmes douées et enthousiastes surgissent parmi les Religieuses Hospitalières du Madawaska. Selon les Soeurs Rhéa Larose et Lucie Morneault, il faut aller de l'avant et donner ce qu'il y a de meilleur à la jeunesse. En 1943, ces deux grandes éducatrices décident de fonder, en collaboration avec Soeur St-Charles, une école d'infirmières à Saint-Basile. Les jeunes filles n'auront donc plus à s'éloigner de leur famille pour suivre le cours de garde-malades.

L'amour de la jeunesse incite aussi Soeur Larose à prendre des moyens pour assurer une éducation intégrale. Au risque de s'attirer, ditelle, " des voyages de briques par la tête ", elle organise la Jeunesse Étudiante Catholique, des concours oratoires, des cercles culturels et, comme ses devancières, des " petites séances musicales et théâtrales ". L'enseignement de la gymnastique, des danses folkloriques et même du ballet est fortement encouragé. Afin de former « un esprit sain dans un corps sain ", Soeur Larose s'avise de faire construire un camp étudiant « là-haut sur la montagne ».

Cette femme débordante d'énergie a aussi le souci d'aider les élèves moins bien doués intellectuellement. Au prix de grands sacrifices, des classes et des cours spéciaux sont mis sur pied afin de favoriser le succès du plus grand nombre possible de jeunes. Les " travaux de goût " ne sont plus maintenant réservés à un groupe choyé. De grands locaux sont aménagés pour l'enseignement de ce que l'on appelle " cours ménager » : couture, tricot, tissage, broderie, cuisine, reliure, cuir repoussé, etc.

Cependant, après la guerre 1939-1945, l'évolution sociale secoue fortement l'oeuvre des Hospitalières au Madawaska. Ces dernières cherchent à s'adapter aux situations nouvelles et à ne pas faire obstacle aux initiatives de groupes désireux d'apporter leurs contributions au développement de la société. En 1946, l'Université St-Louis d'Edmundston ouvre ses portes et accueille les garçons dès leur sortie de 
l'école élémentaire. De plus, les Frères de l'Instruction chrétienne arrivent à Saint-Basile pour assumer l'éducation de la gent masculine de la paroisse. Le pensionnat pour garçons fondé en 1885 n'a plus sa raison d'être et les Hospitalières, conscientes qu'elles ne sont plus nécessaires dans ce champ d'apostolat, décident de le fermer en juin 1947.

Le vide causé par le départ des garçons sera comblé, car Soeur Larose a d'autres projets. Le temps est venu d'assurer aux jeunes filles l'éducation supérieure jusqu'alors réservée aux garçons. En 1949 est fondé le premier collège pour filles dans le nord de la province. En cette même année, Soeur Larose obtient du gouvernement provincial l'autorisation d'ouvrir une École Normale pour religieuses. Grâce à cette initiative, 80 religieuses de diverses congrégations recevront, entre 1949 et 1968 , leur diplôme d'enseignement à Saint-Basile.

Soeur Rhéa Larose songe aussi aux petits qui arrivent en première année sans avoir la préparation requise. Pourquoi ne pas ouvrir une maternelle? Et c'est chose faite en 1954.

Quelques années plus tard, le Collège Maillet étend son champ d'activités en instaurant des nouveaux programmes non encore offerts dans les autres institutions francophones du Nouveau-Brunswick : secrétariat médical et juridique et le baccalauréat en nursing.

Il y a donc de la vie sur la butte du Couvent au cours des années 1950 et 1960. Il semble, par contre, que ce soit alors l'apogée de l'Hôtel-Dieu. En effet, après 1970, la vieille Académie et le jeune Collège Maillet subissent de profondes transformations apportées par les besoins des temps nouveaux. Le phénomène de centralisation dans l'éducation réduit, en 1969, l'Académie à une école élémentaire, ce qui entraîne la fermeture du pensionnat des filles. Quand au Collège qui en 1972 compte 155 étudiantes, il doit accepter sa fusion au Collège St-Louis. C'est le commencement de la fin : en septembre 1979 toutes les élèves sont transférées au campus universitaire d'Edmundston. Ces événements bouleversent certainement les Religieuses Hospitalières de Saint-Joseph. Elles acceptent toutefois ces transformations dans un esprit de foi et d'espérance. Elles savent que les temps ont changé depuis ce message de Soeur Pagé en 1874: "Vous comprenez bien qu'il vous faudra garder l'école encore de longues années».

Plus de cent ans se sont écoulés depuis ce message et l'Académie de Madawaska, devenue École Maillet, appartient toujours à la Congrégation et quelques Hospitalières y enseignent encore. D'autres ont été orientées, par les événements, vers les écoles avoisinantes ou vers l'université. 
Les Hospitalières enseignantes des années 1980 cherchent à découvrir les vues du Seigneur sur leur rôle dans l'éducation. Quels sont les véritables besoins dans ce domaine en cette fin du $20^{\circ}$ siècle ? La réponse à cette question se trouve dans le charisme de leur congrégation énoncé en 1978 :

Nous. Religieuses Hospitalières de Saint-Joseph. sommes appelées à vivre la liberté des enfants de Dieu, en femmes de foi. incarnant la tendresse et la compassion du Christ dans le service de ses membres. spécialement les pauvres. les malades et les plus démunis. en UNION DE CHARITÉ.

Soeur Georgette DESJaRdins. r.h.s.j.. Centre Universitaire St-Louis-Maillet, Edmundston, N.-B. 